\title{
investigación
}

\section{OBJETIVOS Y PERSPECTIVAS DE LA INVESTIGACION EUROPEA EN EL CAMPO DEL HORMIGON}

\author{
Julio Ferry Borges, \\ Dr. Ingeniero $(*)$ \\ 070-12
}

Agradecemos al Profesor Bergström, organizador de la Conferencia sobre Investigación Europea en el Campo del Hormigón (Estocolmo, junio 1981), su amable autorización para publicar en INFORMES este artículo que recoge la contribución del autor a dicha Conferencia.

La traducción del original inglés ha sido realizada por José Pedro Gutiérrez, del IETCC.

\section{RESUMEN}

Se presentan brevemente las necesidades de investigación sobre aspectos fundamentales del cálculo estructural del hormigón, comportamiento estructural (carga monotónica, comportamiento sísmico, situaciones frente al fuego, impacto y fatiga), durabilidad, materiales y tecnología de la construcción y organización de la construcción con hormigón.

Se hace énfasis en la necesidad de generalizar el uso de los conceptos de requisitos y de comportamiento y abordarlos bajo una aproximación probabilista.

Se llama la atención sobre la posibilidad de utilizar la aproximación probabilista de la fiabilidad fuera del campo habitual del comportamiento estructural.

Se recomienda el estudio de los problemas de garantia de calidad, basados en investigaciones sico-sociológicas, para mejorar los métodos que

(*) Director del Laboratorio Nacional de Ingenieria de Lisboa y Presidente del Comité Euro-Internacional del Hormigón. deben emplearse en la organización de las actividades de construcción.

Por último, se comentan las perspectivas a corto y medio plazo de la investigación europea sobre el hormigón.

\section{INTRODUCCION}

Durante las ultimas tres décadas la construcción en hormigón ha experimentado un enorme progreso, que puede expresarse no sólo por el volumen anual de construcción sino también por la mejora de los métodos de cálculo y de las técnicas de construcción.

La producción anual mundial de cemento portland ha aumentado desde cerca de 130 millones de toneladas en 1950 (1) hasta 850 millones de toneladas en 1980 y se espera alcanzar la cifra de 2.000 millones de toneladas a finales de siglo.

Hace treinta años, el cálculo de estructuras de hormigón se basaba en conceptos que no eran satisfactorios desde un punto de vista racional. Las técnicas de construcción estaban limitadas por la calidad del hormigón y del acero; la industrialización y la prefabricación estaban en sus comienzos; la eficacia de la maquinaria de construcción era baja comparada con los niveles medios actuales.

Aunque se construyeron algunas estructuras muy importantes de hormigón armado antes de los años cincuenta, su concepción estaba limitada por las herramientas de cálculo y métodos de construcción disponibles.

Las últimas tres décadas han conducido a un progreso rápido de los métodos experimentales y numéricos de analisis estructural.

Las técnicas experimentales han tenido un rápido desarrollo tanto en ensayos sobre modelos como en la observación del comportamiento estructural. Captadores eléctricos de deformaciones y desplazamientos, conectados a dispositivos automáticos de rastreo, permitieron realizar medidas exactas, rápidas y económicas. Se desarrollaron un gran número de nuevos tipos de instrumentación: escalas simples para la medida de anchos de fisuras y sofisticados registradores de los desplazamientos de estructuras (Fig. 1). Se construyeron grandes instalaciones estáticas y 


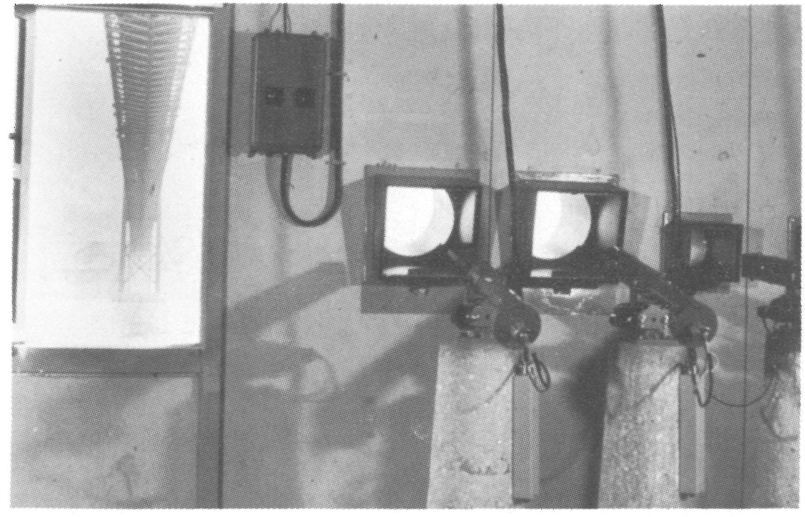

Fig. 1.-Sistema telescópico para la medición continua de desplazamientos.

dinámicas para ensayos estructurales. en Europa, América del Norte y Japón.

Aprovechando el desarrollo exponencial de los computadores, los métodos numéricos basados en el concepto de los elementos finitos, permitieron el análisis elástico de cualquier tipo de sistema estructural (Fig. 2). Los algoritmos de cálculo fueron cada vez más eficientes. Los problemas no-lineales geométricos y físicos se resolvieron fácilmente.
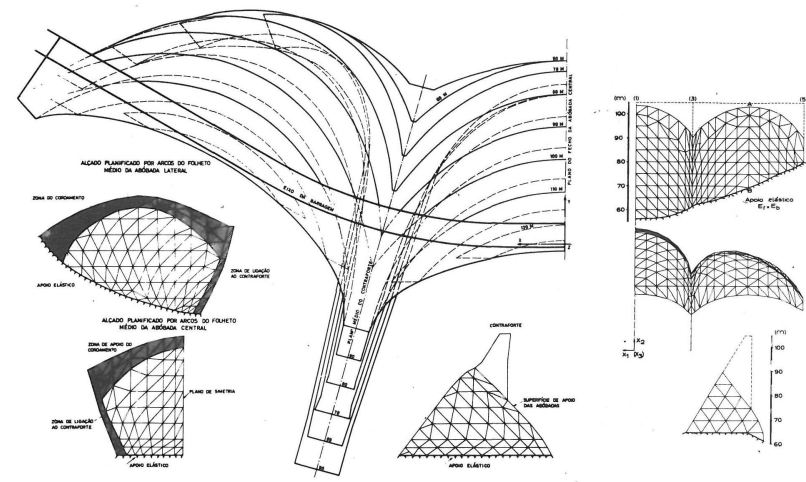

Fig. 2.--Malla de elementos finitos para el estudio de una presa de hormigón multi-bóveda (Presa Aguieira).

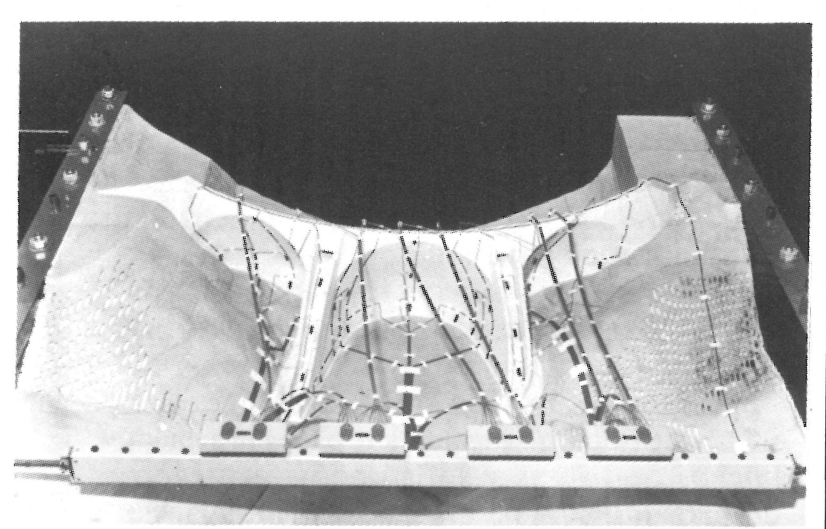

Fig. 3. --Ensayo del modelo de la presa Aguieira.
Actualmente, cualquier estructura que se conciba puede ser analizada, ensayada (Fig. 3) y observada (Fig. 4) fácilmente. Los resultados del cálculo analítico y experimental pueden compararse sistemáticamente con los resultados de las observaciones del comportamiento real. La información sobre el comportamiento de edificios, puentes, presas y otros tipos de estructuras está disponible para ser reunida y transformada en mejores reglas de cálculo.

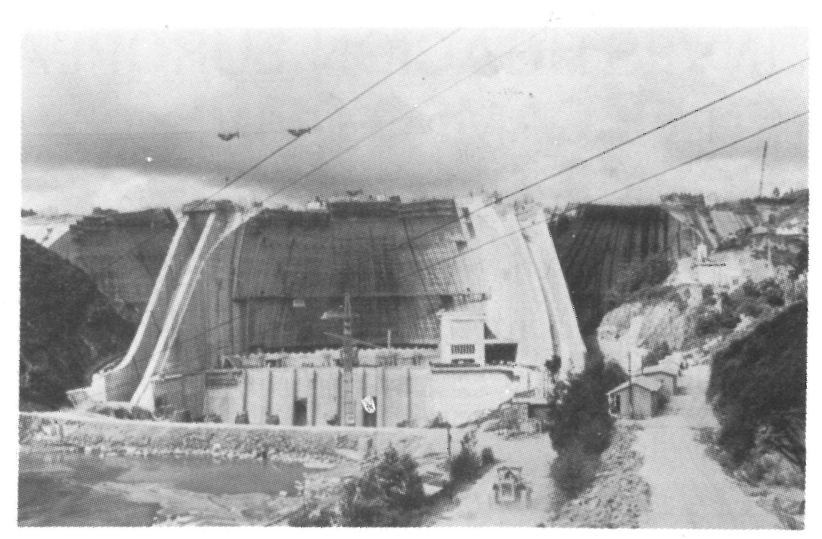

Fig. 4. --La presa de Aguieira en la fase final de construcción.

\section{NECESIDADES DE INVESTIGACION}

\section{Generalidades}

Los gobiernos han empleado diversos métodos para la determinación de prioridades en ciencia y tecnología para la planificación general de los programas científicos y tecnológicos (2).

No se ha conseguido todavía la combinación de las necesidades nacionales en planes regionales conjuntos y su agrupación en planes mundiales, como recomiendan las Naciones Unidas (3).

Hasta el momento, no se han identificado las necesidades de investigación en la construcción con hormigón mediante el empleo de estos marcos operacionales avanzados.

Se ha celebrado una reunión internacional en julio de 1977 en la Universidad de California, en Berkeley, con el propósito específico de identificar las necesidades de investigación en la construcción con hormigón (tratando principalmente la construcción en zonas sísmicas).

En 1978, el Comité Euro-Internacional del Hormigón ha creado un Comité consultivo sobre investigación y aplicación. Este Comité está dando unas directrices importantes al trabajo del CEB. Otras asociaciones internacionales tales como el CIB y la RILEM tienen Comités equivalentes. 
La identificación de las necesidades de investigación técnica, a nivel internacional, parece más fácil de alcanzar mediante las asociaciones internacionales específicas que a través de la iniciativa de los gobiernos.

En este artículo se presenta un punto de vista sobre las necesidades de investigación en la construcción de hormigón, sugiriendo líneas generales de investigación y resaltando áreas de estudio particularmente importantes.

Para obtener una panorámica satisfactoria de las necesidades de investigación en la construcción de hormigón se requiere también cubrir una serie de campos asociados que incidan en todos los tipos de construcción. De esta forma las materias se amplifican y se detectan lagunas importantes.

Los temas que se cubren brevemente a continuación son: conceptos básicos de cálculo, comportamiento estructural, durabilidad, materiales y tecnología de la construcción y organización de la construcción con hormigón.

\section{Conceptos básicos de cálculo}

En el estado actual de evolución del cálculo estructural, los progresos que se esperan principalmente provienen de la mejora de los conceptos y datos básicos.

Durante las tres últimas décadas se ha construido la teoría probabilista de fiabilidad. Ahora es un cuerpo casi completo preparado para su implantación (4). La aplicación cuantitativa de esta teoría implica la existencia de datos sobre acciones, propiedades mecánicas, dimensiones y comportamiento, estando todos los datos modelizados de acuerdo con los conceptos probabilistas. Esta investigación necesaria está bien identificada dentro del Comité Conjunto sobre Seguridad Estructural y se están dando pasos para preparar la documentación general necesaria.

La racionalización del cálculo implica la definición previa de los requisitos y de los criterios de comportamiento. La introducción en los años cuarenta del concepto de estado limite fue un primer paso en esta dirección. Sin embargo, sólo recientemente se ha reconocido la necesidad de profundizar en la definición y cuantificación de las exigencias que cubran todos los aspectos de la construcción, incluyendo los estructurales (5).

Las reglas de cálculo basadas en los ensayos o en procedimientos analíticos, encontrarian su justificación si se exige su cumplimiento. Las prescripciones específicas deberían verse como consideraciones que proporcionan formas de cumplir los requisitos.
La armonización de códigos internacionales, nacionales y regionales parece más fácil de alcanzar en los niveles generales de los conceptos y requisitos que en el nivel específico de las reglas de comprobación. Un ejemplo de cooperación en esta línea lo proporcionan el Instituto Americano del Hormigón, ACI, y el Comité Euro-Internacional del Hormigón, CEB, que crearon un Comité Conjunto sobre criterios de comportamiento («performance criteria»).

La identificación de las necesidades de investigación es uno de los mayores beneficios de la discusión de los requisitos y de su cuantificación mediante criterios de comportamiento. Esto se aplica a los problemas estructurales relacionados con la resistencia y deformabilidad y llega a ser particularmente evidente cuando se discuten requisitos sobre durabilidad, impermeabilidad, resistencia al fuego, etc.

La política de emplear conceptos probabilistas para expresar los criterios de comportamiento, conduce a tener reglas de decisión basadas en las probabilidades de ocurrencia dentro de un intervalo de tiempo dado. Para fijar estas probabilidades deberían considerarse los condicionamientos económicos y sociales $(6$ y 7 ).

Por otra parte, la teoría probabilista de la fiabilidad ofrece un modelo parcial de la realidad, ya que no cubre errores gruesos y negligencias, aspectos destacados a considerar cuando se discute la seguridad.

La garantía de calidad cubre el estudio de todos estos aspectos y debería verse como el marco general donde formular los problemas generales relacionados con el cálculo, construcción y utilización, afectando a todos los participantes en el proceso de construcción.

Este punto de vista conduce a considerar la garantía de calidad como un soporte fundamental sobre el que basar la organización de la industria de la construcción.

\section{Comportamiento estructural}

\section{Generalidodes}

Las teorias de estructuras definen el

comportamiento estructural, esto es, las relaciones entre acciones, solicitaciones, desplazamientos, deformaciones, torsiones, fisuración, rotura, etc., como una función de la geometría de la estructura, las propiedades de los materiales, el tiempo y las condiciones ambientales.

Las estructuras están formadas normalmente por elementos típicos: vigas, columnas, losas, etc. Así, se consideran dos etapas en los estudios estructurales; en la primera se define el 
comportamiento estructural de los elementos; en la segunda, se ensamblan los elementos y se analiza el comportamiento total de la estructura.

El objetivo principal de la teoría del hormigón estructural consiste en definir el comportamiento de los elementos de hormigón, que pueden ser armados, pretensados, parcialmente pretensados o de hormigón en masa.

Las idealizaciones que se adoptan en las teorias del hormigón estructural difieren según los problemas a resolver. Normalmente las teorias se refieren a las situaciones de construcción y utilización. Se han utilizado diferentes aproximaciones cuando se analizan situaciones de riesgo tales como terremotos, fuegos, impactos, vibraciones mantenidas, etc.

Al identificar las necesidades de investigación debe hacerse distinción de los tipos de situaciones.

En muchos casos las situaciones de construcción y utilización pueden describirse mediante cargas monotónicas, despreciando la influencia del tiempo. En consecuencia, este tipo de problema es el que fundamentalmente tratan los códigos básicos estructurales.

\section{Cargas monotóniccas}

Se han estudiado extensamente los problemas estructurales relacionados con las cargas monotónicas. Se han construido teorias que describen el comportamiento; por ejemplo, en el caso de flexión combinada (que incluye los casos extremos de tracción o compresión pura), cortante, torsión, etc. Sin embargo, son fácilles de identificar, incluso en estas teorias, puntos débiles que requieren investigación.

En los códigos actuales, la flexión última se basa en una limitación arbitraria de las deformaciones del acero y del hormigón (9). El establecimiento de estos límites llevó muchos años de discusiones dentro del CEB. Si la flexión última se define como el valor resistente máximo, el problema se resolvería buscando este máximo, en la hipótesis de que los diagramas tensión-deformación del hormigón y del acero tienen ramas descendentes. Se debería considerar la aleatoriedad de estos diagramas y se debería dar una interpretación probabilista a este máximo. Debería incluirse información sobre la deformación última del acero en tracción, confinamiento del hormigón, recubrimientos, pandeo de barras a compresión, etcétera, para obtener respuestas completamente satisfactorias.

De modo similar, los modelos que permiten definir la resistencia a cortante basados en la analogía de celosía de Mörsch, han sido mejorados recientemente mediante la consideración de ángulos variables para la fisuración inclinada. Los limites del campo de variabilidad de estos ángulos son también arbitrarios y deberían introducirse criterios mejorados para definir la resistencia a cortante y para tener en cuenta la participación del hormigón comprimido y de las barras longitudinales, transversales y oblicuas.

Aunque las necesidades de investigación son fáciles de identificar en los problemas básicos citados anteriormente, las decisiones recientes del CEB se han concentrado en otras áreas, tales como: efectos térmicos, cálculo de losas, inestabilidad de estructuras, vigas de gran canto, punzonamiento, comportamiento en servicio, detalles de armado, etcétera. La elección de estos problemas es el resultado de la disponibilidad de investigadores dispuestos a abordarlos. La aproximación ideal de la relación coste/beneficio para la determinación de prioridades $y$, en consecuencia, la definición de actividades de investigación, es en la actualidad dificil de conseguir.

El CEB está investigando mediante simulación en áreas básicas tales como: comportamiento multiaxil del hormigón en masa, comportamiento dependiente del tiempo, idealización de elementos armados bidimensionales, etc.

Esto está de acuerdo con un movimiento general en la dirección de una más profunda comprensión de los mecanismos del hormigón estructural (10). En este movimiento se identifican dos aproximaciones.

La primera aproximación trata de estudiar el comportamiento bi y tridimensional por medio de formulaciones mejoradas de elementos finitos, basadas en ecuaciones constitutivas prácticas que permiten seguir la evolución no-lineal hasta rotura, considerando incluso efectos del tiempo y alternancia de acciones.

La segunda aproximación es más fundamental. Su objetivo es establecer las ecuaciones generales basadas en la mecánica de fracturas, fractura plástica y teorías endocrónicas, que modelen el hormigón de una forma refinada. Hasta el momento las ecuaciones derivadas por esta vía no son simples, lo que parece ser inherente a la complejidad y variedad de los fenómenos físicos modelizados.

\section{Comportamiento sísmico}

La ingeniería sísmica es un campo de investigación muy activo (11). Aunque se han alcanzado grandes progresos mediante la investigación llevada a cabo en muchos países, existe todavía un largo camino por recorrer para lograr métodos de calculo sísmico completamente satisfactorios cuantitativamente. 
Del lado de las acciones, el cálculo sísmico comprende la definición de la sismicidad de las regiones, lo que incluye mecanismos de generación, leyes de propagación, amplificación local, interacción suelo-estructura, etc. La idealización probabilista de las acciones sísmicas incluye modelos descriptivos y de ocurrencia.

Aunque los conocimientos en todos estos problemas progresan rápidamente, no ha sido posible todavía alcanzar un consenso internacional sobre la idealización práctica de las acciones sísmicas para los casos usuales.

La Asociación Internacional de Ingenieria Sísmica ha intentado una clasificación de conceptos mediante la creación de un Comité que estudie los conceptos básicos de los códigos sísmicos. Un primer borrador de este trabajo fue presentado en septiembre de 1980 en la Conferencia Mundial de Estambul y un segundo borrador se presentó en la reunión del Comité Conjunto sobre Seguridad Estructural que se celebró en Trondheim en 1981.

Hay un considerable desfase entre el conocimiento científico y técnico en ingeniería sísmica y el empleo de códigos en paises diferentes (12). En la actualidad todavía ocurren daños muy importantes en regiones donde hay códigos modernos.

Un movimiento importante para mejorar esta situación se debe al Consejo de Aplicación Tecnológica, por la publicación de su «Intento de Recomendaciones para Cálculo Sísmico, ATC-3»y otros documentos en la misma línea han contribuido mucho al progreso en este área (13).

Además, el comité Euro-Internacional del Hormigón, CEB, ha publicado en marzo de 1980 el primer borrador de un apéndice sísmico para el Código Modelo CEB-FIP (14). El documento final será presentado en la próxima Asamblea General que se celebrará en Munich en abril de 1982.

El documento del CEB identifica las exigencias y criterios de comportamiento que deben emplearse en el cálculo sísmico, recomienda métodos de cálculo que deben aplicarse en diferentes casos (edificios regulares y no regulares) y especifica detalles de armado asociados con diferentes niveles de ductilidad. Este documento es el resultado de una cooperación mundial amplia.

Sin embargo, los criterios actuales de cálculo sísmico no son todavía completamente satisfactorios. Esto se debe principalmente a la inexactitud de la definición de la deformabilidad última de los elementos estructurales sometidos a ciclos alternos de alto nivel, análogos a aquellos debidos a la acción sísmica.

La cuantificación de la probabilidad de fallo debería calcularse mediante la convolución de las probabilidades de las acciones y sus desplazamientos resultantes que sobrepasan limites dados y las probabilidades de que las estructuras sufran estos desplazamientos sin pérdida de su capacidad resistente en situaciones normales. La información sobre las distribuciones últimas es todavía escasa (6).

La protección y mitigación de riesgos sísmicos es un problema multidisciplinar complejo. Una discusión en términos generales, incluyendo los problemas de armonización de códigos, rediseño y readecuación de estructuras dañadas, tuvo lugar en octubre de 1981 en Lisboa, durante el Segundo Seminario sobre Construcción en Regiones Sísmicas, organizado por la Comisión Económica para Europa.

\section{Comportamiento al fuego}

La protección de estructuras de hormigón contra el fuego se ha basado en los resultados de los ensayos de fuego realizados en estaciones experimentales, o sobre la experiencia obtenida en fuegos reales. Ha faltado una protección racional contra el fuego.

Las investigaciones recientes realizadas en diferentes universidades europeas han mostrado la posibilidad de emplear métodos numéricos, particularmente técnicas de elementos finitos, para estudiar la evolución del comportamiento del hormigón estructural con la temperatura y el tiempo. Mediante una idealización adecuada de los efectos de la temperatura sobre las propiedades mecánicas de los materiales, es posible seguir la evolución de una estructura durante un fuego.

Como en otras situaciones, la mejora del cálculo estructural frente al fuego depende de la identificación de los requisitos y criterios de comportamiento. En este caso el problema es particularmente complicado debido a las relaciones entre medidas estructurales y medidas de lucha contra el fuego.

Las soluciones óptimas implican un compromiso entre estos aspectos tan diferentes.

Las necesidades de investigación sobre protección contra el fuego incluyen, por ejemplo, una definición mejor de la evolución de la temperatura durante los fuegos, la idealización de las variaciones de las propiedades térmicas y mecánicas del hormigón y acero con la temperatura y el tiempo y la comparación entre los resultados numéricos y experimentales sobre el comportamiento estructural durante los fuegos.

Varias Asociaciones Internacionales están trabajando en este campo: la RILEM se está ocupando de los efectos de la temperatura sobre 
las propiedades mecánicas de los materiales utilizados en la construcción; el CIB está señalando posibles aproximaciones conceptuales a la seguridad estructural en el cálculo de ingeniería frente al fuego; y el CEB está preparando un borrador para un apéndice del Código Modelo que cubra el cálculo de estructuras de hormigón en situaciones de fuego. Se espera que la cooperación de estas Asociaciones, que también incluye a la FIP, conduzca a resultados prácticos útiles.

En un futuro cercano, la ISO aprovechará todos estos esfuerzos para mejorar la normalización existente.

\section{Impacto $y$ fatiga}

Existe una variedad tal de situaciones de riesgo ("hazard scenarios») que conduce a problemas de impacto que su clasificación resulta muy discutible.

Es normal considerar que los impactos y cargas impulsivas conducen a perforaciones, daños locales y colapso estructural total.

El comportamiento estructural está íntimamente relacionado con el proceso de disipación de energía durante el impacto.

La clasificación de los problemas que provocan fatiga es aún más difícil. Hay una variedad de mecanismos de fallos que van desde unos pocos ciclos alternos de alto nivel hasta fatiga de ciclos bajos y altos. Las acciones pueden idealizarse mediante ciclos deterministas, procesos aleatorios o una combinación de ambos.

Los conceptos probabilistas deberían utilizarse ampliamente para definir las propiedades mecánicas de fatiga, daños acumulativos y efectos del proceso de carga.

Aunque los problemas de fatiga son importantes en algunos tipos de construcciones (puentes, grúas, torres, mástiles, plataformas marítimas) rara vez lo son en la construcción con hormigón. Sin embargo, el problema merece estudiarse y una buena oportunidad para identificar las necesidades de investigación será el "Coloquio sobre Fatiga de Estructuras de Hormigón y Acero» de la IABSE, que se celebrará en Lausana en marzo de 1982.

De una forma análoga, los problemas de impacto y las necesidades de investigación en este área se discutirán durante el simposio inter-asociaciones «Estructuras de hormigón sometidas a cargas de impacto e impulsivas» que se celebrará en Berlín en junio de 1982.

\section{Durabilidad}

En la actualidad, las exigencias de durabilidad se introducen simplemente mediante la indicación de que los requisitos estructurales deben mantenerse a lo largo de la vida prevista de la estructura y, en consecuencia, las estructuras deberían diseñarse y mantenerse de modo que tengan una fiabilidad adecuada (4). La complejidad y variedad de los problemas de durabilidad no es consistente con este punto de vista unificado. La durabilidad debería tratarse como un requisito por separado, identificando primero los diferentes procesos de deterioro $y$, en consecuencia, imponiendo su limitación.

En estructuras de hormigón, los procesos de deterioro más importantes relacionados con el material hormigón son: disolución de hidratos de calcio, carbonatación del hormigón, formación de estringita, hielo y deshielo y fatiga por tensiones cíclicas. Los procesos de deterioro de la armadura son: oxidación, corrosión del acero por cloro, corrosión bajo tensión, fatiga por tensiones cíclicas. Los problemas de fatiga se mencionaron cuando se discutió el comportamiento estructural y se omitirán aquí.

El propósito principal de los estudios de durabilidad consistiría en identificar las variables principales que controlan los procesos de deterioro y establecer las leyes que idealizasen aquellos procesos. Estas idealizaciones deberían tratarse con las técnicas normales de la fiabilidad probabilista, es decir, con métodos de nivel II.

Existe el convencimiento de que esta forma de tratar los problemas es fácil de expresar y difícil de poner en práctica.

En los problemas estructurales normales, las ecuaciones de estado límite incluyen como variables básicas: acciones, propiedades mecánicas de los materiales, dimensiones y parámetros del modelo. En los procesos de deterioro las variables básicas serían: composición química y proporción de la mezcla, propiedades físico-químicas de los materiales, dimensiones, tiempo y parámetros del modelo. Al igual que en los problemas estructurales, estas variables deberían expresarse mediante distribuciones de probabilidad.

\section{Materiales y tecnología de la construcción}

La evolución anterior muestra que la mayor contribución al progreso de la construcción en general y de la construcción de hormigón en particular, deriva de materiales mejores y técnicas de construcción más eficientes. Los objetivos generales que deben alcanzarse son: economía, velocidad de construcción y eficiencia. 
La tendencia en la construcción en hormigón ha sido la utilización de hormigones de resistencias cada vez mayores.

Esta tendencia puede crear problemas debidos a la menor ductilidad relativa de los hormigones de muy alta resistencia. Estos problemas pueden manifestarse no sólo en elementos sometidos a tensiones altas de compresión, sino también en el caso de acciones indirectas; las fisuras anchas son más difíciles de evitar en hormigones de alta resistencia que en los hormigones normales.

Los hormigones con fibras o resinas están encontrando un campo creciente de aplicaciones. Su tecnología y propiedades se están estudiando en varios laboratorios de investigación. El empleo estructural del hormigón ligero se limita a las regiones que se han concentrado en esta tecnología. El empleo del hormigón ligero no se ha extendido tan rápidamente como cabría esperar hace pocos años.

El progreso en la inyección de tendones pretensados debiera derivarse del uso generalizado de las técnicas de vacío y de nuevos materiales y técnicas de inyección.

La tendencia a emplear acero de armar de resistencia muy alta (con tensiones de plastificación mayores de $60 \mathrm{MPa}$ ) está decreciendo. Las tecnologías de producción de barras de hormigón armado y acero de pretensado parecen estar estabilizadas. La posición es completamente diferente en lo que concierne a la tecnología del armado, donde se espera una revolución debida a la automatización.

La construcción de estructuras de hormigón in situ está progresando debido al uso intensivo de encofrados perfeccionados, que incluyen perfiles especiales de aluminio, paneles de madera de alta calidad, moldes de plástico y equipos cada vez más potentes de bombeo y mezcla. La industrialización y la prefabricación juegan un papel importante en la construcción con hormigón. Sin embargo, en los países donde primeramente comenzó la prefabricación no parece necesario incrementar el número de factorías de prefabricación.

En el futuro debe prestarse más atención a los problemas de mantenimiento y rehabilitación, lo que resulta necesario para ampliar la vida útil de los edificios existentes por encima de los límites inicialmente previstos. Para estudiar este problema va a celebrarse en Lisboa en marzo/abril de 1982 un Simposio Conjunto ASTM/CIB/RILEM sobre el Concepto del Comportamiento en Edificios, que cubrirá los avances en el desarrollo de esta área y su aplicación a la rehabilitación.

En obras públicas, particularmente en puentes, se espera un incremento del empleo del pretensado

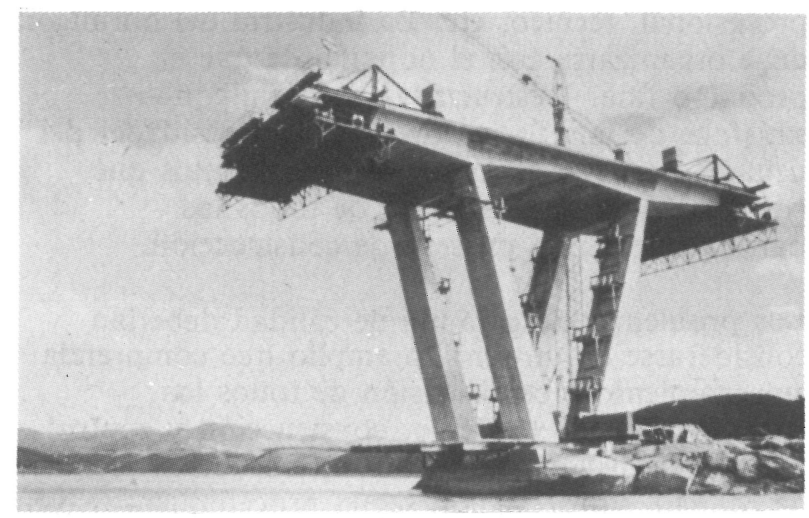

Fig. 5. --Puente de hormigón parcialmente pretensado en construcción.

parcial (Fig. 5). Aunque las reglas de cálculo cubren el pretensado parcial, la aplicabilidad de estas reglas deberían ponerse en forma de ejemplos y difundirse, para facilitar el cálculo de estructuras parcialmente pretensadas.

Los problemas específicos de construcción en zonas sísmicas deberían recibir una atención especial. Las experiencias pasadas muestran que los sucesos sísmicos son en muchas regiones las causas más importantes de desastres, debido a fallos de construcción (Fig. 6).

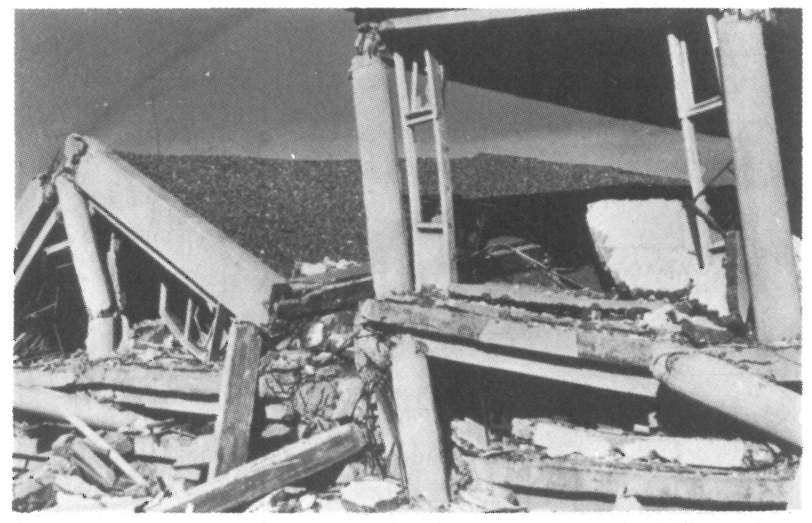

Fig. 6.--Estructura de hormigón armada dañado por el terremoto de El Asnam de octubre de 1980.

\section{Organización de la industria de la construcción con hormigón}

La organización y desarrollo de la industria de la construcción en Europa es muy diferente de un país a otro. Existen, sin embargo, principios generales que se aplican a todas las etapas del proceso constructivo. Estos principios deberían discutirse extensamente y establecerse claramente. $\left({ }^{*}\right)$.

La organización de la industria del hormigón tiene muchos aspectos: financiero, económico,

(*) N. de R. - En la Sección CUADERNOS DE INFORMES del número extraordinario 333-34-35-36 se publicó un trabajo al respecto, que corresponde a la Referencia (8) de las citadas por el autor al final de este artículo. 
profesional, técnico, etc. La industria del hormigón debe organizarse con el objetivo de que el producto final - estructuras de hormigónsatisfaga de una forma óptima las necesidades del usuario, respetando los condicionamientos que corresponden a los intereses de todos los participantes en el proceso de construcción.

Los problemas de garantía de calidad deberían considerarse en un sentido amplio que comprenda: entrenamiento y cualificación de todos los participantes en el cálculo, construcción y control de la construcción; verificación, inspección, control de calidad y observación según procedimientos bien definidos; definición de tareas y responsabilidades; información y procedimientos de comunicación y coordinación; preparación de documentos; códigos, normas, manuales, etc. (Fig. 7).

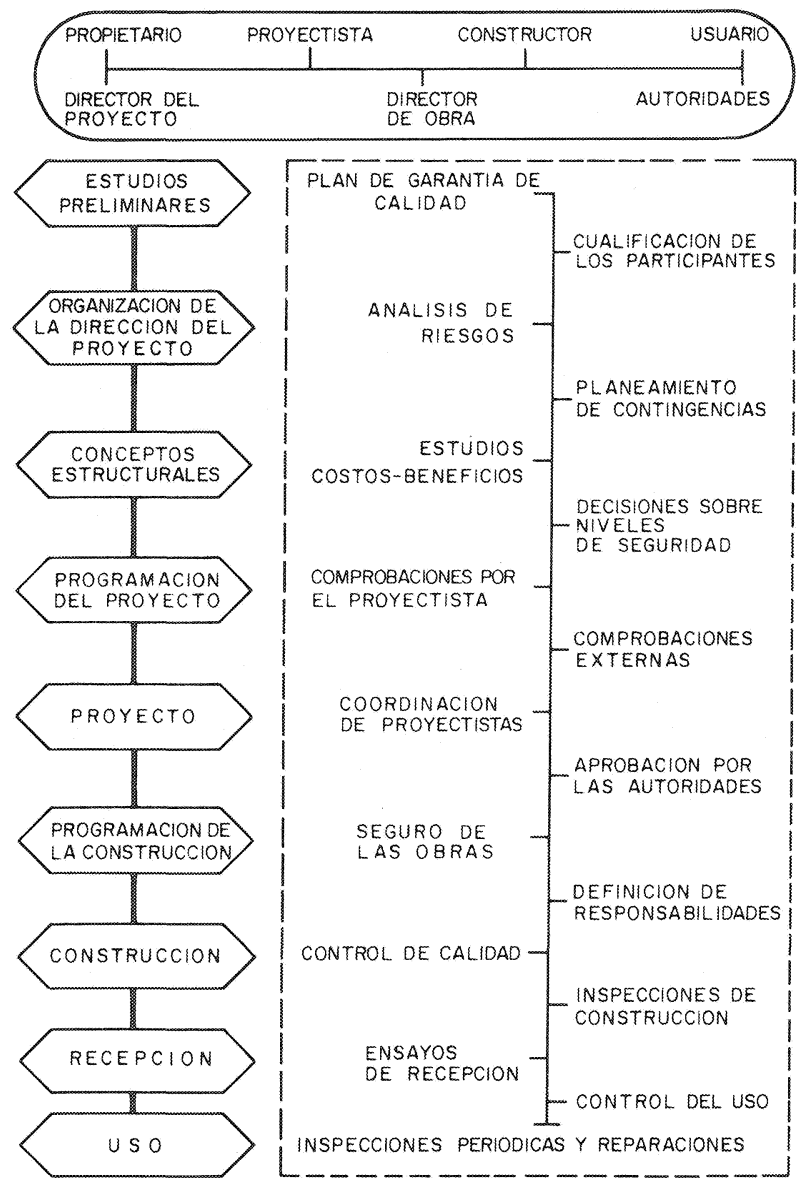

Fig. 7.--Participantes, actividades y aspectos de la garantia de calidad relacionados con el proceso de construcción.

La base científica para soportar el estudio de varios de estos problemas debería ampliarse a la economía, sociología, sicología y otras ciencias que tratan del comportamiento humano. Hasta el presente, el número de estudios en este área es muy limitado y las decisiones están siendo tomadas sin base científica.
Se espera que la sico-sociología juegue un papel importante en el estudio de la garantía de calidad. $\mathrm{Su}$ aplicación debería extenderse a campos afines tales como la definición de las necesidades del usuario y la evaluación de soluciones.

\section{PERSPECTIVAS DE LA INVESTIGACION SOBRE EL HORMIGON EN EUROPA}

Para satisfacer las necesidades humanas, se espera un incremento de la industria mundial del hormigón en los campos de la edificación, obras públicas, y otros tipos de construcción. Cuando existen beneficios es fácil justificar la necesidad de un incremento paralelo de la investigación sobre el hormigón. Sin embargo, la situación actual en Europa muestra un panorama desfavorable. En diversos países los fondos gubernamentales destinados a la investigación se han reducido debido a las dificultades económicas globales. En otros institutos de investigación, que dependen de los fondos proporcionados por las industrias de los materiales y de la construcción, no se han visto incrementados sus fondos proporcionalmente al incremento de las ventas en estas industrias.

Consecuentemente, el potencial humano científico dedicado a la investigación en ingeniería civil y, en particular, a la investigación del hormigón, se ha mantenido estable si es que no ha disminuido.

Como en otras ramas de actividad, la productividad de la investigación necesita mejorarse. Esto debería alcanzarse mediante una cooperación intensiva entre los institutos de investigación. El medio más efectivo de alcanzar esta cooperación es a través de las Asociaciones Internacionales. Esta posición se comprende bien en Europa. El Comité Euro-Internacional del Hormigón está jugando un papel activo mediante la identificación de las necesidades de investigación, coordinando investigaciones, revisando conocimientos existentes $y$, particularmente, elaborando información técnica avanzada. Esta información técnica se publica en forma de códigos modelos, manuales, informes sobre el estado del arte, etc., documentos todos que son discutidos en profundidad.

Otras Asociaciones Internacionales tales como ACI, CIB, FIP, IABSE, RILEM, etc., contribuyen activamente al progreso de la construcción con hormigón.

La efectividad de las Asociaciones Internacionales implica su coordinación. Para las Asociaciones que tienen sus sedes centrales en Europa, ésta se alcanza a través de un Comité de Enlace. Para estudiar problemas de interés común se han creado Comités Conjuntos, por ejemplo, el Comité Conjunto sobre Seguridad Estructural. 
Existen muchos aspectos en los que esta coordinación debería mejorarse. La voluntad de que así sea depende de todos los participantes en las actividades internacionales.

Durante las últimas décadas, la cooperación internacional ha dado un gran paso adelante debido principalmente a la supresión práctica de las barreras del idioma. Esta tendencia debería mantenerse mediante una futura reducción de los nacionalismos técnicos.

Las agencias internacionales y las organizaciones económicas regionales juegan un papel importante para estimular la investigación. Las organizaciones regionales e internacionales para la normalización deberían aprovechar el trabajo realizado por las asociaciones internacionales.

Debido a la complejidad del nacimiento y funcionamiento de las asociaciones internacionales, su amalgamamiento no parece ser la forma más efectiva para incrementar su eficacia. Sin embargo, su proliferación es también extremadamente peligrosa, pues conduciría a organizaciones con una visión demasiado estrecha y muy difícil de coordinar.

\section{REFERENCIAS}

(1) - World Cement Market in Figures, Cembureau, The European Cement Association, Paris 1973.

(2) - Method for Priority Determination in Science and Technology, Science Policy Studies and Documents, n. ${ }^{\circ} 40$, UNESCO, Paris 1978.

(3) - World Plan of Action for the Application of Science and Technology to Development, United Nations, New York 1971 .

(4) - General Principles on Reliability for Structural Design, Joint-Committee on Structural Safety, Report, Volume 35, Internationational Association for Bridge and Structural Engineering, Zürich 1981.

(5) - ISO Guide for the Preparation of Performance Standards in Building, DP 6241, ISO TC 59/SC 3 Document n. ${ }^{\circ} 68$, April 1978.

(6) - Borges, J. Ferry - Structural Safety, course 101, Laboratorio Nacional de Engenharia Civil, Lisbon, 1971.

(7) - Bosshard, W. - Structural Safety - Matter of Decision and Control, IABSE Surveys, International Association for Bridge and Structural Engineering, Periodica, May 1979.

(8) - General Principles on Quality Assurance for Structures, Joint-Committee on Structural Safety, Report, Volume 35, International Association for Bridge and Structural Engineering, Zürich $1981\left(^{(*)}\right.$.

\section{CONCLUSIONES}

El enorme valor económico de las construcciones en hormigón justifica una gran actividad de investigación que debería cubrir los aspectos generales aplicables a todo tipo de construcción y los aspectos específicos de las construcciones de hormigón.

Las necesidades de investigación en diferentes áreas: métodos de cálculo, comportamiento estructural, durabilidad, materiales y tecnología de la construcción y organización de la industria de la construcción, están bien identificadas. El tamaño y complejidad de la mayoría de estos problemas justifica que su solución sea obtenida a través de una cooperación internacional amplia. La productividad total de investigadores, institutos, países e incluso regiones que trabajan separadamente es mucho más pequeña que la productividad que se obtendría de su labor coordinada. Hasta el presente, la cooperación internacional está limitada en muchos casos por los límites continentales. Esto justifica el porqué este artículo se ha centrado en la investigación europea sobre el hormigón. Se siente la necesidad de organizar una cooperación internacional más amplia.
(9) - CEB-FIP Model Code for Concrete Structures, Euro-International Committee for Concrete, Bulletin d'Information, 124/125, Paris 1978.

(10) - Advanced Mechanics of Reinforced Concrete, Colloquium Introductory Report, International Association for Bridge and Structural Engineering, Volume 33, Delft 1981.

(11) - International Directory of Universities and Institutions Engaged in Earthquake Engineering Research, International Association for Earthquake Engineering, Tokyo, 1976.

(12) - Earthquake Resistant Regulations. A World List. International Association for Earthquake Engineering, Tokyo, August 1980.

(13) - Tentative Provision for the Development of Seismic Regulations for Buildings, ATC 3-06, Applied Technology Council, National Bureau of Standards Special Publication 510, Washington 1978.

(14) - Seismic Design of Concrete Structures, Draft of an Appendix to the CEB-FIP Model Code, Euro-International Committee for Concrete, Bulletin d'Information n. ${ }^{\circ}$ 133, Paris, March 1980.

(*) N. de R. - Este trabajo se publicó en la Sección CUADERNOS DE INFORMES del número extraordinario 333-34-35-36. 